\title{
Health economic measures in schizophrenia research
}

PAUL MCCRONE

\section{Background It is essential in economic evaluations of schizophrenia interventions that all relevant costs are identified and measured appropriately. Also of importance is the way in which cost data are combined with information on outcomes.}

\begin{abstract}
Aims To examine the use of health economics in evaluations of interventions for schizophrenia.
\end{abstract}

Methods A review of the key methods used to estimate costs and to link costs and outcomes was conducted.

Results Costs fall on a number of different agencies and can be short term or long term. Cost-effectiveness analysis and cost-utility analysis are the most appropriate methods for combing cost and outcome data.

Conclusions Schizophrenia poses a number of challenges for economic evaluation.

Declaration of interest P.M. has received speaker fees from Eli Lilly and Janssen-Cilag.
In evaluating interventions to treat schizophrenia it is clearly important to assess the clinical and social effects of the intervention and to measure these appropriately. It will often be desirable to include a number of outcome measures, ranging from those with a relatively narrow perspective (e.g. symptom measures) to those which are more holistic (e.g. quality of life measures). Ideally, we would hope that decision makers would only favour interventions which have proven efficacy. Although good evidence is certainly required for new treatments or services, some of those in existence probably do not have as firm an evidence base.

Evidence that an intervention is effective does not in itself mean that it should be implemented. All interventions require the use of resources (money, time, equipment, etc.), and these will inevitably be limited in their supply and able to be used to provide care in other ways (whether for people with schizophrenia or with asthma, cancer, etc.). Resource scarcity and competing demands for these resources means that the costs of interventions for schizophrenia need to be assessed in addition to outcomes.

\section{MEASURING COSTS}

Costs occur when a productive activity takes place that necessitates the use of scarce resources that could be used for some other purpose. Economists describe this as an opportunity cost. The time that psychiatrists, community mental health nurses, psychologists, social workers and others spend in providing care for people with schizophrenia could be spent with other patient groups, and hence a cost arises. Unpaid care (from families/friends) will also frequently be used, and this also has an opportunity cost.

The focus of economic analyses is often on the final figure (in pounds, dollars, euros, etc.), but this is simply a proxy measure for care inputs (and in schizophrenia these will be many) that a patient receives. It is generally incorrect to regard costs as outcomes; rather they are a representation of inputs that produce outcomes. Some of the impacts of interventions for schizophrenia can be measured in monetary units, for example in-patient admissions, if a reduction is the main aim of the intervention, as with crisis resolution teams.

To estimate the cost of schizophrenia care it is necessary to identify all the resources required to provide this care and also those resources that are subsequently affected. The costs of treatment with atypical antipsychotics, for example, include not only the specific drug costs but also the professional time required to administer and monitor treatment plus the cost of other care (such as in-patient stays and outpatient appointments) if these may change as a result of the drug treatment.

A common costing perspective in economic evaluations is to include all health service costs. This is the preferred view of the UK's National Institute for Health and Clinical Excellence (NICE), whose remit is to offer guidance on the spending of $\mathrm{Na}$ tional Health Service finances. In schizophrenia care even this will be inadequate, as interventions which are successful (e.g. in reducing positive symptoms) could alter the use a patient makes of social care, education and criminal justice services. Costs to families and friends in terms of the unpaid care (but representing costly lost opportunities) provided and also the potential value of patient time spent in contact with services could also be included.

The Client Service Receipt Inventory (CSRI; Beecham \& Knapp, 2001) is the most commonly used questionnaire to measure service costs for people with schizophrenia. The CSRI is usually completed through interviews with patients, although case notes and information from carers can be used, and some versions can be completed by the patients themselves. The information collected with the CSRI, or a similar measure, can be combined with appropriate unit cost information (e.g. Curtis \& Netten, 2006) to generate service costs.

\section{Costing systems of care}

Several studies have assessed the economic impact of alternative approaches to delivering schizophrenia care. In a review of home-based care (assertive community 
treatment and crisis interventions) Burns $e t$ al (2001) identified 22 studies that had included an economic component. It was encouraging that many of these did take a relatively comprehensive approach to costing and that this had improved compared with an earlier review (McCrone \& Weich, 1996).

Broader studies have attempted to cost the impact of schizophrenia at a national and even international level. Making comparisons between countries presents difficulties given variations in the methodologies used (Andlin-Sobocki \& Rössler, 2005). Differences in price levels generally (and healthcare prices in particular), the supply of specific services and the structure of mental health services will all affect service costs, as will variations in patient need. Nevertheless, it can still be informative to examine the contribution that specific services make to overall service costs.

In-patient care has consistently been the most costly service since deinstitutionalisation began. Knapp et al (2004) reported that it accounts for between 28 and $94 \%$ of direct healthcare costs in schizophrenia, whereas medication usually accounts for less than $15 \%$ of costs. One exception was in Nigeria where medication accounted for $62 \%$ of costs, reflecting the much higher drug costs relative to cost of inpatient care in low- and middle-income countries. This is a vivid reminder that cost findings in one area cannot be simply translated to another. The supply of services will naturally have a major impact on utilisation and therefore costs. In a five-country comparison of schizophrenia care (Table 1) costs were particularly low in a Spanish city where there was limited availability of day or residential care (Knapp et al, 2002).

\section{Costs of specific interventions}

Economic evaluations have been conducted of a wide range of interventions for schizophrenia (McCrone \& Weich, 1996; Byford et al, 2003). Most tend to concentrate on healthcare costs, with some also including social care and criminal justice service costs. Very few evaluations have assessed the impact that interventions have on informal care costs, an important omission as many community-based interventions could well increase the necessity for care from family members or friends.

Another neglected cost is patient time spent using services. In economics generally, time is valued using information

Table I Comparative 3-month costs of schizophrenia care in five European cities'

\begin{tabular}{lccccc}
\hline & \multicolumn{5}{c}{ Cost in 1996, $£$} \\
\cline { 2 - 6 } Service & Amsterdam & Copenhagen & London & Santander & Verona \\
\hline Hospital in-patient & 320 & 5772 & 3659 & 1456 & 2705 \\
Hospital out-patient & 236 & 376 & 139 & 0 & 627 \\
Day care & 2293 & 774 & 1091 & - & 1650 \\
Community services & 551 & 241 & 1086 & 90 & 459 \\
Residential care & 764 & 130 & 749 & - & 298 \\
All care & 4112 & 7460 & 6771 & 1444 & 5760 \\
\hline
\end{tabular}

I. Adjusted for differences in patient characteristics. From Knapp et al (2002).

on an individual's wages, but the majority of patients in contact with specialist services are not in work. However, this does not mean that the value of time is zero time spent on other activities will still have to be forgone in order to use services. This might be relevant when evaluating assertive community treatment, which is principally targeted at patients who are 'difficult to engage'. Although it is probable that engagement problems are usually a result of the clinical features of schizophrenia, some patients might be engaged in other activities that they value and which limit the time which they wish to spend accessing services (even if this is to the detriment of their mental health).

Cost information is also relatively limited concerning the period before patients receive care for schizophrenia. The duration of untreated psychosis (DUP) has been reported to be up to 1-2 years (McGlashan, 1999). Case-note narratives and studies of pathways into care suggest that this period is characterised by contacts with criminal justice services, visits to accident and emergency departments and interruptions to employment. All of these consequences incur costs, and interventions to reduce the DUP should reduce these, but to date no study has calculated this effect. Further discussion of the DUP is provided by Singh (2007) in this supplement.

Most economic (and clinical) evaluations have relatively short time scales. The consequence of this is that the longer-term cost impact of improved, or worsened, outcomes is neglected. If an intervention is successful then it is highly likely that this will have a long-term impact on the use and cost of services, especially for people with a long-term condition such as schizophrenia where some of the care inputs required have particularly high costs. However, the extent of cost savings as a result of successful interventions is unclear. Engagement with services might be enhanced, which could actually increase long-term costs. However, it is probably more likely that effective care will reduce the need for crisis services - and in particular emergency admissions - if effectiveness means fewer and less severe relapses.

\section{LINKING COSTS TO OUTCOMES}

The measurement of costs associated with interventions for schizophrenia forms only one component of an economic evaluation. The synthesis of information on costs with data on effectiveness is the essence of economic evaluation. This is illustrated in Fig. 1 where costs (should) depend on patient needs and influence outcomes. There are different forms of economic evaluation and these differ according to how outcomes are measured.

\section{Cost-minimisation analysis}

There are some situations where the outcomes of different services are known $a$ priori, and therefore only costs need to be measured. If outcomes are identical then the least-cost option is the preferred one. However, given the variety of possible interventions for schizophrenia (typical and atypical antipsychotics, psychological therapies, early intervention, etc.) only rarely will outcomes be known with confidence beforehand.

\section{Cost-effectiveness analysis}

Cost-effectiveness analysis combines cost information with data on a single conditionspecific outcome measure, for example symptomatology or social functioning. This 


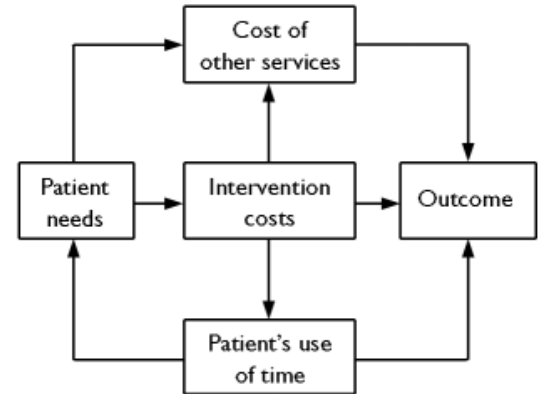

Fig. I Relationship between patient needs, costs and outcomes.

form of analysis can have particular relevance for clinicians. However, it is limited given that schizophrenia affects patients in many ways. This is a common limitation of most clinical evaluations, which generally specify a primary outcome measure.

\section{Cost-consequences analysis}

This is a more general case of cost-effectiveness analysis that reflects the broad impact that interventions have. Rather than one single outcome measure being used, a number of different measures are considered. Economic evaluations of interventions could combine cost-effectiveness and costconsequences analyses, with the former conducted using the primary clinical outcome measure and secondary measures simply reported alongside the cost findings.

\section{Cost-utility analysis}

This is similar to cost-effectiveness analysis but uses a generic measure of outcome. This enables interventions in diverse areas (e.g. cancer and stroke) to be compared in terms of cost-utility with those for schizophrenia. The most common outcome measure in this form of analysis is the quality-adjusted life-year (QALY). Having a generic measure of outcome is of particular importance for those making recommendations across diverse clinical areas. In the UK, NICE bases many of its findings on the cost per QALY achieved by different interventions.

Quality-adjusted life-years are a composite measure of the amount of time spent in a particular healthcare state and the quality of life experienced during that time. The latter is measured on a scale from 0 (death) to 1 (full health). The most common quality of life measure used in economic evaluations is the EuroQoL-5D (EQ-5D; Williams, 1995). This consists of five domains (mobility, self-care, usual activities, pain/discomfort and depression/anxiety) and respondents state whether they have no problem, some problems or major problems for each domain. This results in distinct health states to which 'utility' or quality of life scores (on a $0-1$ scale) are attached. Such scores have been derived from a general population survey where people were asked to compare health states with full health (Dolan et al, 1995).

To date, cost-utility analysis has been used infrequently in studies of care for schizophrenia. Most QALY measures (such as the EQ-5D) focus largely on physical health problems and there are questions about the sensitivity of QALY measures to changes in mental health states (Chisholm et al, 1997). However, there have been a small number of attempts to use the QALY method in studies of schizophrenia care (e.g. Rosenheck et al, 1998; Sevy et al, 2001) and more work in this area would be useful.

\section{Cost-benefit analysis}

Cost-benefit analysis compares the costs of a particular service with the outcomes achieved also measured in monetary terms. If the outcomes in monetary terms exceed the costs then the service is efficient. One of the very earliest economic evaluations of community mental health services in Madison (Wisconsin, USA) used this method, with earnings from work used as the main outcome measure (Weisbrod et al, 1980). The breadth of ways in which interventions for schizophrenia might affect patients suggests that cost-benefit analysis will rarely be appropriate.

\section{ANALYSIS AND INTERPRETATION OF ECONOMIC RESULTS}

If a new intervention for schizophrenia is compared with usual care then a number of results could occur. It would be appropriate to adopt an intervention if it results in lower costs than existing care and better outcomes. The intervention should also be favoured if outcomes are no different but costs are reduced or if costs are the same and outcomes are improved. Usual care would be the preferred option if the results were the opposite way round. However, it is unclear whether or not a new intervention should be adopted if outcomes are better but costs are higher. In effect this becomes a value judgement, with the key question being whether or not the increased costs are justified by the level of improved outcomes. Similarly, there is ambiguity about the appropriateness of an intervention if it saves money but is less effective. Interestingly, there appear to be few studies in this category. Interventions with significantly better outcomes than comparators will frequently reduce costs (especially those associated with admissions).

Many economic evaluations actually have uncertain results. For example, both the UK700 study (Byford et al, 2000) and the PRiSM Psychosis Study (McCrone et al, 1998), which compared the costs and outcomes of intensive and standard care services for people with psychosis, found no statistically significant differences between costs and outcomes.

To address such uncertainty, it is becoming common practice to link cost and outcome data from cost-effectiveness and cost-utility analyses using incremental cost-effectiveness ratios (ICERs). An ICER is defined as the ratio between the difference in costs between two interventions and the difference in outcomes. The ICER then allows us to state the cost that is incurred for an intervention to produce one unit of outcome more than a comparator. An ICER is only informative if one intervention is both more effective and more costly than its comparator (otherwise the intervention or the comparator would be the dominant option). Relating incremental costs to incremental outcomes suggests that a unit improvement in outcome (e.g. a 1point decrease in score on the Brief Psychiatric Rating Scale or the Positive and Negative Syndrome Scale) has a meaning to decision makers. The latter may not hold and so more tangible outcome measures might be preferred (e.g. measuring outcome in terms of number of clinically important changes or number of people above or below a particular clinical cut-off point).

One of the problems with ICERs is that they rely on point estimates of costs and outcomes and yet there will be uncertainty around both of these. This has led economists to produce cost-effectiveness acceptability curves. These show the probability that one intervention is more cost-effective than another for alternative values (thresholds) placed on a unit improvement in outcome.

\section{CONCLUSIONS}

Since the 1980s there has been a substantial increase in the number of studies that have examined the costs associated with 
schizophrenia and economic evaluations of specific interventions for this disorder. The quality of studies has generally improved but there are still limitations. In particular, recent methodological developments in health economics generally need to be applied to multi-site studies to generate evidence from the large samples that these studies can provide. Schizophrenia affects the lives of patients in many ways and it is essential that outcome measures used in economic evaluations reflect this. What is clear is that the costs of care should be measured comprehensively and over an adequate period. Costs also need to be linked to outcomes appropriately. The most suitable methods for doing this appear to be cost-effectiveness and cost-utility analyses.

\section{REFERENCES}

Andlin-Sobocki, P. \& Rössler, W. (2005) Cost of psychotic disorders in Europe. European Journal of Neurology, 12 (suppl. I), 74-77.

Beecham, J. \& Knapp, M. (200I) Costing psychiatric interventions. In Measuring Mental Health Needs (2nd edn) (ed. G. Thornicroft), pp. 200-224. Gaskell

Burns, T., Knapp, M., Catty, J., et al (200I) Home treatment for mental health problems: a systematic review. Health Technology Assessment, 5, I-139.

PAUL McCRONE, PhD, Department of Health Service and Population Research, King's College London, Institute of Psychiatry, De Crespigny Park, London SE5 8AF, UK. Email: p.mccrone@iop.kcl.ac.uk

Byford, S. Fiander, M., Torgerson, D. J., et al (2000) Cost-effectiveness of intensive $v$. standard case management for severe psychotic illness: UK700 case management trial. British Journal of Psychiatry, I76, 537-543.

Byford, S., McCrone, P. \& Barrett, B. (2003) Developments in the quantity and quality of economic evaluations in mental health. Current Opinion in Psychiatry 16, 703-707.

Chisholm, D., Healey, A. \& Knapp, M. R. J. (1997) QALYs and mental health care. Social Psychiatry and Psychiatric Epidemiology, 32, 68-75.

Curtis, L. \& Netten, A. (2006) Unit Costs of Health and Social Care. Personal Social Services Research Unit.

Dolan, P., Gudex, C., Kind, P., et al (1995) A Social Tariff for EuroQol: Results from a UK General Population Survey. Centre for Health Economics, University of York.

Knapp, M., Chisholm, D., Leese, M., et al (2002) Comparing patterns and costs of schizophrenia care in five European countries: the EPSILON study. Acto Psychiatrica Scandinavica, 105, 42-54.

Knapp, M., Mangalore, R. \& Simon, J. (2004) The global costs of schizophrenia. Schizophrenia Bulletin, 30 279-293.

McCrone, P. \& Weich, S. (1996) Mental health care costs: paucity of measurement. Social Psychiatry and Psychiatric Epidemiology, 31, 70-77.
McCrone, P., Thornicroft, G., Phelan, M., et al (1998)

Utilisation and costs of community mental health services. PRiSM Psychosis Study. 5. British Journal of Psychiatry, I73, 391-398.

McGlashan, T. H. (1999) Duration of untreated psychosis in first episode schizophrenia: marker or determinant of course, Biological Psychiatry, 46, 899-907.

Rosenheck, R., Cramer, J., Xu, W., et al (1998)

Multiple outcome assessment in a study of the costeffectiveness of clozapine in the treatment of refractory schizophrenia. Health Services Research, 33, 1237-1261.

Sevy, S., Nathanson, K., Schechter, C., et al (200I) Contingency valuation and preferences of health states associated with side effects of antipsychotic medications in schizophrenia. Schizophrenia Bulletin, 27, 643-652.

Singh, S. P. (2007) Outcome measures in early psychosis. Relevance of duration of untreated psychosis. British Journal of Psychiatry, 19I (suppl. 50), s58-s63.

Weisbrod, B. A., Test, M. A. \& Stein., L. I. (1980) Alternative to mental hospital treatment: II. Economic benefit-cost analysis. Archives of General Psychiatry, 37 400-405.

Williams, A. (1995) The Role of the EuroQoL Instrument in QALY Calculations. Centre for Health Economics, University of York. 